\title{
ERRATUM
}

\section{Ultra stable self-assembled monolayers of N-heterocyclic carbenes on gold}

Cathleen M. Crudden, J. Hugh Horton, Iraklii I. Ebralidze, Olena V. Zenkina, Alastair B. McLean, Benedict Drevniok, Zhe She, Heinz-Bernhard Kraatz, Nicholas J. Mosey, Tomohiro Seki, Eric C. Keske, Joanna D. Leake, Alexander Rousina-Webb and Gang Wu

Nature Chemistry 6, 409-414 (2014); published online 23 March 2014; corrected after print 10 April 2014.

In the version of this Article originally published, J. Hugh Horton should also have been denoted a corresponding author, with the e-mail address: jhh@queensu.ca. This error has now been corrected in the online versions of the Article.

\section{ADDENDUM}

\section{Quantitative sequencing of 5-formylcytosine in DNA at single-base resolution}

Michael J. Booth, Giovanni Marsico, Martin Bachman, Dario Beraldi and Shankar Balasubramanian

Nature Chemistry 6, 435-440 (2014); published online 23 March 2014; corrected after print 6 May 2014.

The raw data including sequencing files (fastq format), processed data including counts of modified bases for each library preparation (reduced, oxidative and simple bisulfite) and the estimation of significant sites as reported in the paper, together with some explanation and a summary of the experimental and analytical protocols, are available here: http://www.ncbi.nlm.nih.gov/geo/query/acc.cgi?acc=GSE56572 